\title{
ON SINGULAR ELLIPTIC EQUATION WITH SINGULAR NONLINEARITIES, HARDY-SOBOLEV CRITICAL EXPONENT AND WEIGHTS
}

\section{Mohammed El Mokhtar Ould El Mokhtar and Zeid I. Almuhiameed}

Abstract. This article is devoted to the existence and multiplicity to the following singular elliptic equation with singular nonlinearities, Hardy-Sobolev critical exponent and weights:

$$
\begin{cases}-\Delta u-\mu \frac{u}{|x|^{2}}=\frac{|u|^{p-2} u}{|x|^{s^{2}}}+\lambda \frac{u}{|x|^{\alpha}}|u|^{-\beta}, & x \in \Omega, \\ u>0 & x \in \Omega, \\ u=0 & x \in \partial \Omega .\end{cases}
$$

where $\Omega$ is a smooth bounded domain in $\mathbb{R}^{N}(N \geqslant 3), 0 \in \Omega, \lambda>0,0 \leqslant \mu<\bar{\mu}_{N}:=$ $(N-2)^{2} / 4, p=2^{*}(s)=2(N-s) /(N-2)$ with $0<s<2$ is the critical Hardy-Sobolev critical exponent, $0 \leqslant \alpha<N(p-1+\beta) / p, 0<\beta<1$ and $2<p \leqslant 2^{*}:=2 N /(N-2)$ is the critical Sobolev exponent.

By using the Nehari manifold and mountain pass theorem, the existence of at least four distinct solutions is obtained.

Mathematics subject classification (2010): 35J66, 35J55, 35B40.

Keywords and phrases: Singular elliptic equation, singular nonlinearities, Hardy-Sobolev critical exponent, Nehari manifold, variational method.

\section{REFERENCES}

[1] A. BAhri, J. M. Coron, On a nonlinear elliptic equation involving the critical Sobolev exponents: the effect of the topology of the domain, Comm. Pure Appl. Math. 41 (1988), 253-294.

[2] M. BoucheKiF, A. Matallah, On singular nonhomogeneous elliptic equations involving critical Caffarelli-Kohn-Nirenberg exponent, Ric. Mat. 58 (2009) 207-218.

[3] H. Brezis, L. Nirenberg, Positive solutions of nonlinear elliptic equations involving critical Sobolev exponents, Comm. Pure. Appl. Math. 36 (1983), 437-477.

[4] K. J. BROWN, Y. ZHANG, The Nehari manifold for a semilinear elliptic equation with a sign changing weight function, J. Differential Equations 2 (2003) 481-499.

[5] D. CAO, S. PENG, A note on the sign-changing solutions to elliptic problems with critical Sobolev exponent and Hardy terms, J. Differential Equations 193 (2003) 424-434.

[6] J. CHEN, Existence of solutions for a nonlinear PDE with an inverse square potential, J. Differential Equations 195 (2003) 497-519.

[7] J. Q. CHEN, Multiple positive solutions of a class of nonlinear elliptic equations, J. Math. Anal. Appl. 295 (2004), 341-354.

[8] J. I. DíAZ, Nonlinear Partial Differential Equations and Free Boundaries, Vol I. Elliptic Equations, Pitman, London, 1985.

[9] L. DING, C. L. TANG, Existence and multiplicity of solutions for semilinear elliptic equations with Hardy terms and Hardy-Sobolev critical exponents, Appl. Math. Lett. 20 (2007), 1175-1183.

[10] I. Ekeland, N. GHoussoub, Selected new aspects of the calculus of variations in the large, Bull. Amer. Math. Soc. 39 (2002) 207-265.

[11] M. Ghergu, V. Radulescu, Nonlinear PDEs: Mathematical Models in Biology, Chemistry and Population Genetics, Springer Monographs in Mathematics, Springer Verlag, Heidelberg, 2011. 
[12] D. KANG, On the elliptic problems with critical weighted Sobolev-Hardy exponents, Nonlinear Anal. 66 (2007) 1037-1050.

[13] D. S. KANG, On the quasilinear elliptic equations with critical Sobolev-Hardy exponents and Hardyterms, Nonlinear Anal. 68 (2008), 1973-1985.

[14] D. S. KAng, S. J. PEng, Positive solutions for singular critical elliptic problems, Appl. Math. Lett. 17 (2004), 411-416.

[15] D. S. KAng, S. J. Peng, Solutions for semilinear elliptic problems with critical Sobolev-Hardy exponents and Hardy potential, Appl. Math. Lett. 18 (2005), 1094-1100.

[16] D. Kang, S. Peng, Positive solutions for singular elliptic problems, Appl. Math. Lett. 17 (2004) 411-416.

[17] Z. LiU, P. HAN, Existence of solutions for singular elliptic systems with critical exponents, Nonlinear Anal. 69 (2008) 2968-2983.

[18] M. Schechter, W. M. Zou, On the Brezis-Nirenberg problem, Arch. Ration. Mech. Anal. 197 (2010), 337-356.

[19] S. TERRACINI, On positive entire solutions to a class of equations with singular coefficient and critical exponent, Adv. Differential Equations 1 (1996) 241-264. 